\title{
Four-Wave Mixing in Landau-Quantized Graphene
}

\author{
Jacob C. König-Otto,,,,++ Yongrui Wang, ${ }^{\text {If }}$ Alexey Belyanin, ${ }^{\text {, }}$ Claire Berger, ${ }^{\S}$ \\ Walt A. de Heer, ${ }^{\S}$ Milan Orlita,,$\| \perp$ Alexej Pashkin, ${ }^{\dagger}$ Harald Schneider,${ }^{\dagger}$ Manfred \\ Helm, ${ }^{\dagger, t}$ and Stephan Winnerl ${ }^{\dagger}$ \\ $\dagger$ Helmholtz-Zentrum Dresden-Rossendorf, P.O. Box 510119, 01314 Dresden, Germany \\ $\$$ Technische Universität Dresden, 01062 Dresden, Germany \\ ITTexas AEM University, College Station, Texas 77843-4242, USA \\ $\S$ Georgia Institute of Technology, Atlanta, GA 30332, USA \\ ||LNCMI, CNRS-UGA-UPS-INSA, 38042 Grenoble, France \\ $\perp$ Institute of Physics, Charles University in Prague, 12116 Prague, Czech Republic \\ E-mail: j.koenig-otto@hzdr.de
}

\section{Abstract}

For Landau-quantized graphene, featuring an energy spectrum consisting of a series of nonequidistant Landau levels, theory predicts a giant resonantly-enhanced optical nonlinearity. We verify the nonlinearity in a degenerate time-integrated four-wave mixing (FWM) experiment in the midinfrared spectral range, involving the Landau levels $L_{-1}, L_{L}$ and $L_{1}$. A rapid dephasing of the optically induced microscopic polarization on a timescale shorter than the pulse duration $(\sim 4 \mathrm{ps})$ is observed, while a complementary pump-probe experiment under the same experimental conditions reveals a much longer lifetime of the induced population. The FWM signal shows the expected field dependence with respect to lowest order perturbation theory for low fields. Saturation sets in for fields above $\sim 6 \mathrm{kV} / \mathrm{cm}$. Furthermore, the resonant behavior and the order of magnitude of the third-order susceptibility are in agreement with theoretical calculations.

\section{Keywords}

graphene, four-wave mixing, nonlinear optics, Landau-quantization

The linear dispersion of graphene results in highly nonlinear optical properties in a wide spec- tral range from $\mathrm{THz}$ frequencies to visible light. The third-order nonlinear optical response has been investigated theoretically, both on a semiclassical and a fully quantum mechanical basis ${ }^{1,2}$. In the latter case, in particular the four-wave mixing (FWM) process is addressed. Experimentally FWM signals have been observed in the near-infrared spectral range ${ }^{3-5}$. At THz frequencies, however, only pump-probe signals, but no FWM signals have been found ${ }^{6}$. When a magnetic field is applied perpendicular to the graphene layer the linear dispersion of graphene breaks up into a series of non-equidistant Landau levels ${ }^{7}$. This offers the possibility to resonantly enhance the nonlinear-optical response and to tune the resonance frequency by adjusting the strength of the magnetic field. Recently, a giant nonlinearoptical response has been predicted for Landauquantized graphene ${ }^{8,9}$. In this letter we present the first experimental investigation of this effect by studying transient-degenerate FWM. To this end, the $\mathrm{LL}_{-1} \rightarrow \mathrm{LL}_{0}$ and $\mathrm{LL}_{0} \rightarrow \mathrm{LL}_{1}$ transitions are excited resonantly with radiation at $19 \mathrm{THz}$ $(78 \mathrm{meV})$. The experimental findings are in good agreement with our theoretical calculations based on the density-matrix formalism.

A multilayer epitaxial graphene sample $(\sim 50$ layers) produced by thermal decomposition of $\mathrm{SiC}$ on the $\mathrm{C}$-face of $4 \mathrm{H}-\mathrm{SiC}^{10}$ is used in the experi- 


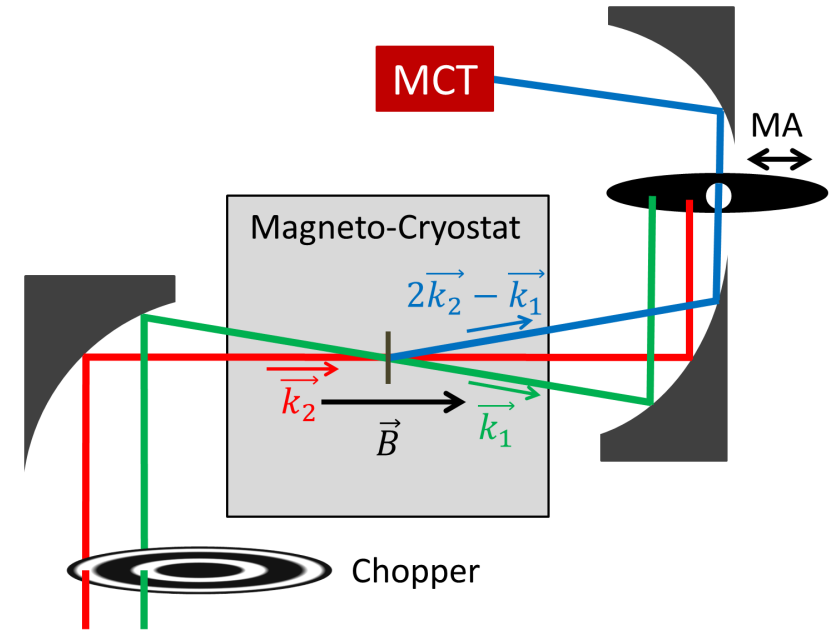

Figure 1: Experimental setup: The movable aperture (MA) is used for selecting the the FWM signal in direction $2 \vec{k}_{2}-\vec{k}_{1}$ or to measure a pump-probe signal in $\vec{k}_{1}$ direction with the MCT detector.

ments. The majority of layers is slightly n-doped and only the layers at the interface to $\mathrm{SiC}$ exhibit a stronger doping and are therefore transparent at the used photon energy of $78 \mathrm{meV}$ and a magnetic field of $4.5 \mathrm{~T}^{11}$. The sample is kept in a split-pair superconducting magnet and the sample temperature is held at $10 \mathrm{~K}$ in a cold helium gas atmosphere. The core of the experimental setup is depicted in Figure 1. The laser pulses from the freeelectron laser FELBE (photon energy of $\sim 78 \mathrm{meV}$ ) are split into two separate paths and are focused with an off-axis parabolic mirror onto the sample inside the magneto cryostat. A time delay $\Delta \mathrm{t}$ between the two pulses can be controlled with a motorized delay stage (not shown). The beams in direction $\vec{k}_{1}, \vec{k}_{2}$ and the FWM signal in direction $2 \vec{k}_{2}-\vec{k}_{1}$ are all collimated by a second parabolic mirror. A movable aperture is used to select, which beam is detected by the nitrogen cooled mercurycadmium-telluride (MCT) detector. The signals are amplified with a lock-in amplifier using a double modulation technique based on the modulation of both incident beams by a dual-slot chopper plate.

If the sample is brought into a magnetic field of around $4.5 \mathrm{~T}$ with a direction orthogonal to the surface, the band structure of graphene breaks up into a system of Landau levels and the $\mathrm{LL}_{-1} \rightarrow$ $\mathrm{LL}_{0}$ and $\mathrm{LL}_{0} \rightarrow \mathrm{LL}_{1}$ transitions become resonant with the photon energy of $78 \mathrm{meV}$. For the almost
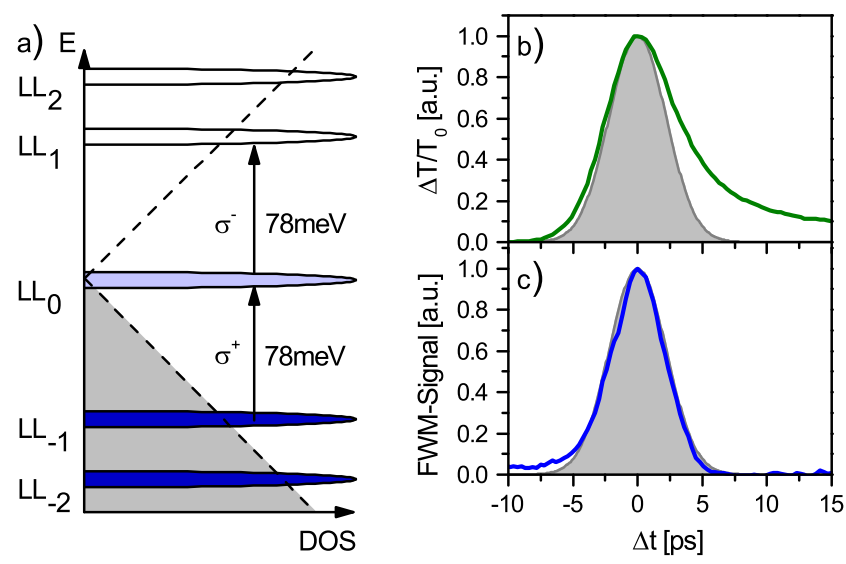

Figure 2: a) The magnetic field of $4.5 \mathrm{~T}$ leads to a Landau-quantization in the graphene sample, whereas the transition from $\mathrm{LL}_{-1}\left(\mathrm{LL}_{0}\right)$ to $\mathrm{LL}_{0}\left(\mathrm{LL}_{1}\right)$ is resonant to the photon energy of $78 \mathrm{meV}$. b) The transient change in population is recorded by a degenerate pump-probe experiment, measuring the transient change of transmission of $\vec{k}_{1}$ (field: $6 \mathrm{kV} / \mathrm{cm}$ ) caused by the absorption of $\vec{k}_{2}$ (field: $12 \mathrm{kV} / \mathrm{cm}$ ). c) The microscopic polarization is probed by detecting the FWM signal $2 \vec{k}_{2}-\vec{k}_{1}$ at the same incident fields. The grayish curves show the autocorrelation of the Laser pulses calculated from spectra.

intrinsic graphene layers $\mathrm{LL}_{-1}$ is fully occupied, $\mathrm{LL}_{0}$ close to half filling and $\mathrm{LL}_{1}$ is completely empty. In detail, we expect the filling of the zeroth Landau-level to be in the order of 0.52 at this magnetic field, based on transmission measurements with circular polarized light. In this work both incident pulses are linearly polarized in the same direction. Thus, the excitation of both transitions is possible as indicated in Figure 2 a). The transient change in transmission $\Delta \mathrm{T} / \mathrm{T}_{0}$ of the sample due to strong optical excitation is depicted in Figure $2 \mathrm{~b}$ ). For this kind of measurement the movable aperture is set to transmit the $\vec{k}_{1}$ beam and the transient change in transmission of this beam caused by the absorption of photons from the $\vec{k}_{2}$ beam is recorded. The pump-probe signal features a fast decay and a slower component in the order of several tens of picoseconds. Recent studies already gave first insight into the population dynamics of this system ${ }^{12-15}$. In particular a rapid (faster than pulse duration of $4 \mathrm{ps}$ ) depopulation of an optically pumped level via Auger scattering has been 
observed $^{15}$. This Coulomb process leads to a fast thermalization of electrons. The slower timescale of the decay is attributed to cooling of the electronic system via phonon scattering.

While the pump-probe signal corresponds mostly to Pauli blocking and reveals the dynamics of the excited population, the FWM signal is sensitive to the induced polarization in the sample. The decay of the microscopic polarization is governed by both the relaxation of the population and by pure dephasing processes. In our experiment, we trace the process where the $\vec{k}_{1}$ beam induces a polarization in the sample. The $\vec{k}_{2}$ beam creates, after the time delay $\Delta \mathrm{t}$, a polarization grating and a part of it is diffracted under momentum and energy conservation bounding conditions into direction $2 \vec{k}_{2}-\vec{k}_{1}$. Figure $2 \mathrm{c}$ ) depicts the FWM signal at the very same experimental conditions as for the pump-probe signal in Figure 2 b). Comparing both types of signals one can see some clear differences. The FWM signal is basically symmetric apart from a small artifact, namely a baseline offset at negative delay times, which stems from pump-probe signals that are scattered as stray light into the detector. Note, that we chose to invert the time delay $\Delta \mathrm{t}$ for the FWM signal in Figure 2 c).to satisfy the inverted time logic of the two experiments. Whereas the beam in $\vec{k}_{2}$ direction is considered as fixed in our pump-probe measurements, contrastingly, in the FWM experiment the beam in $\vec{k}_{1}$ direction defines the time zero. The FWM signal is only present during the temporal overlap of the pulses, indicating that the dephasing time is considerably shorter than the temporal resolution of our experiment set by the pulse duration of around $\sim 4 \mathrm{ps}$. This result, together with the previous detailed study of the population dynamics ${ }^{15}$, suggests that Auger processes within the $\mathrm{LL}_{-1}$, $\mathrm{LL}_{0}$ and $\mathrm{LL}_{1}$ subset of Landau levels cause a rapid dephasing of the microscopic polarization.

Next, we investigate the dependence of the FWM process on the electric fields of the beams in $\vec{k}_{1}$ and $\vec{k}_{2}$ direction. The electric field of the generated FWM signal can be estimated from the induced third-order polarization, which depends on the third-order nonlinear surface (2D) susceptibility $\chi^{(3)}$ (in SI units), as:

$$
\left|\overrightarrow{\mathrm{E}}_{F W M}\right| \simeq \frac{\omega}{2 c}\left|\chi^{(3)} \mathrm{E}^{2}\left(\vec{k}_{2}\right) \mathrm{E}\left(\vec{k}_{1}\right)\right|
$$

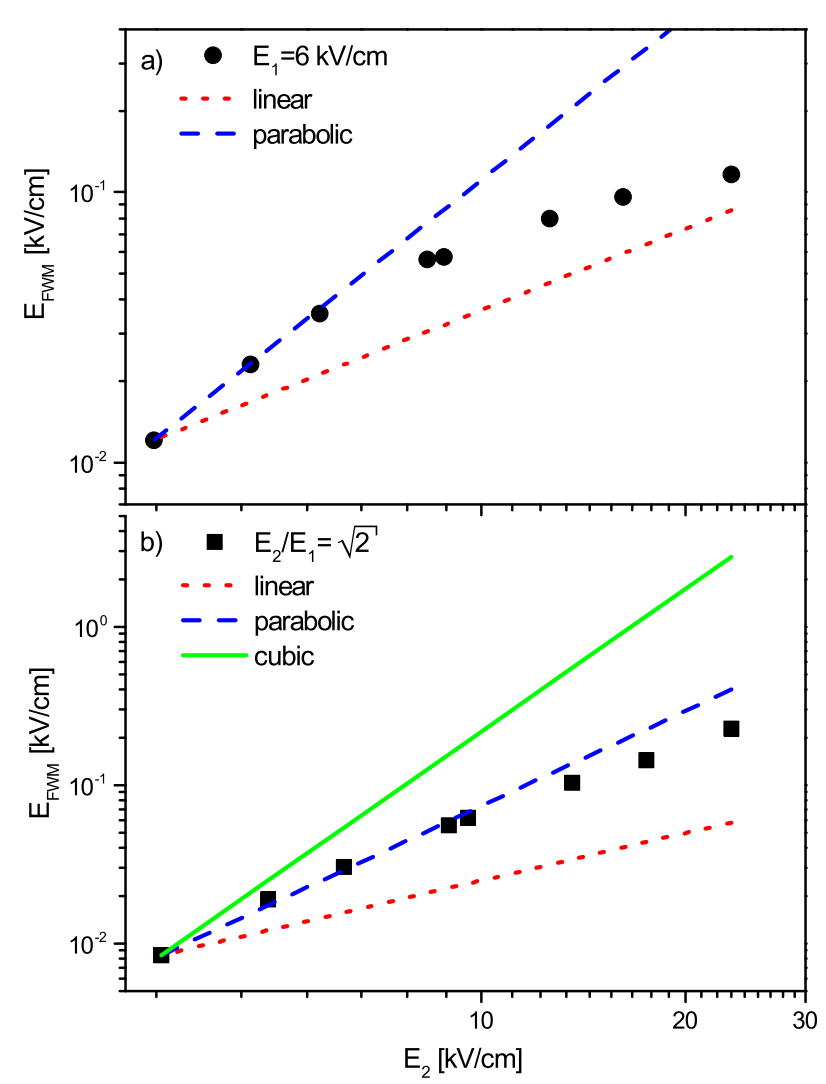

Figure 3: Saturation behavior of the four wave mixing signal: a) The field of beam one is kept at $6 \mathrm{kV} / \mathrm{cm}$, while the field from beam two is varied. b) Both fields are varied while keeping a constant ratio of 2 .

Here $\omega$ is the frequency and $\mathrm{E}\left(\vec{k}_{1}\right)$ and $\mathrm{E}\left(\vec{k}_{2}\right)$ the fields of the incident beams, and $c$ the velocity of light. The surface susceptibility $\chi^{(3)}$ for this process is a function of the magnetic field and the photon energy itself. We will have a closer look on this in the next section. Eq 1 indicates that the FWM field scales linearly the field of the beam in direction $\vec{k}_{1}$ and quadratically with the field of the beam in direction $\vec{k}_{2}$. Thus, if the field of both beams is tuned simultaneously, the FWM field should scale cubically. For a variety of different field combinations of the two incident beams FWM transients were recorded and the maxima of the transients were extracted. The incident peak fields were determined from the measured power, spot size and pulse duration. To determine $\mathrm{E}_{F W M}$ one has to consider the losses at the movable aperture and the calibration of the MCT detector additionally. Note that the inaccuracy of this procedure has no influ- 
ence on the the determination of the scaling behavior of the FWM field with the incident fields. The quantitative values of the FWM field, however, are expected to be accurate only within one order of magnitude. The FWM peak fields are plotted in Figure 3 as a function of the peak field of the beam in direction $\vec{k}_{2}$ beam. Figure 3 a) shows the field dependence when the field of the $\vec{k}_{1}$ beam is kept constant at $6 \mathrm{kV} / \mathrm{cm}$ and only the field of the $\vec{k}_{2}$ beam is varied. Linear and quadratic power laws are indicated by the dotted red and blue dashed line, respectively. The three lowest data points are in agreement with a quadratic dependence. Note that in the case of a pump-probe signal one would only expect a linear scaling of the signal. This, together with the different temporal shapes of the signals (cp. Figure 2), allows one to clearly identify the FWM signal and distinguish it from potential stray pump-probe signals. Nevertheless, a clear saturation is noticeable in the measured field regime. A deeper analysis of saturation effects is beyond the scope of this work. However, in ref 9 saturation fields are calculated for FWM processes with a different level scheme in Landau-quantized graphene under continuous excitation. Despite the differences of the processes evaluated in this work and in ref 9, the reported saturation behavior can serve as an estimation for the order of magnitude of the saturation field. For a magnetic field of $4.5 \mathrm{~T}$ and a dephasing time of $190 \mathrm{fs}$ (this value is discussed later in this letter) the saturation field is in the order of $4 \mathrm{kV} / \mathrm{cm}$, which is qualitatively consistent with the experiment, where a deviation of the square root dependence is observed for fields higher than $6 \mathrm{kV} / \mathrm{cm}$.

In accord with the consideration that two photons of the beam in $\vec{k}_{2}$ direction interact with one photon of the beam in $\vec{k}_{1}$ direction it is reasonable to choose a ratio of the two fields of $E_{2} / E_{1} \approx \sqrt{2}$. The strength of the FWM signal for this constant ratio is depicted Figure $3 \mathrm{~b}$ ). Again the straight lines represent different power laws. The data points do not follow the green line that represents the expected cubic dependence of this case. However, the dependence is found to be superquadratic for the low fields. The observed saturation is not surprising. From Figure 3 a) one can see that at the measured fields already the parabolic dependence on $E_{2}$ saturates. Saturation naturally occurs faster if both fields are varied. It was not possible to achieve a sufficient signal-to-noise ratio at lower fields to clearly demonstrate the cubic dependence.

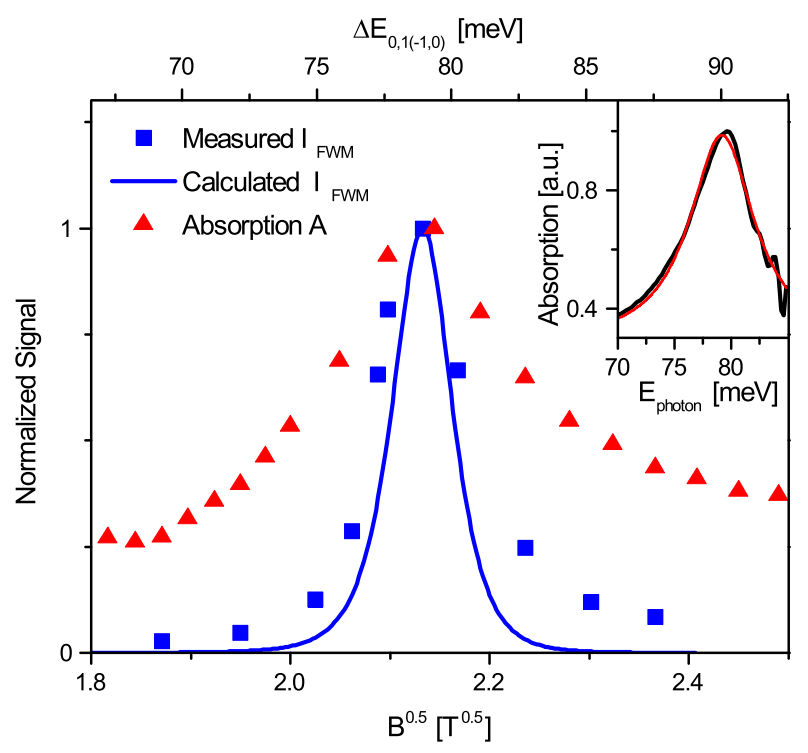

Figure 4: Linear absorption (red triangles)and maxima of FWM mixing signals (blue squares) for different magnetic fields. The field of beam two is $12 \mathrm{kV} / \mathrm{cm}$ and beam one $5.3 \mathrm{kV} / \mathrm{cm}$. The blue line shows the calculated magnetic field dependency of the $\chi^{(3)}$ process. The inset shows a Lorentzian fit to the linear absorption spectra at $4.5 \mathrm{~T}$ to determine the line broadening.

In the following, the resonant behavior of the FWM signal is studied by measuring transients at different magnetic fields, while keeping the photon energy fixed. In Figure 4 the peak FWM intensities are plotted on a $\sqrt{B}$-scale, i.e. a scale that is linear in the energy of the optical transition $\mathrm{LL}_{-1} \rightarrow \mathrm{LL}_{0}$ and $\mathrm{LL}_{0} \rightarrow \mathrm{LL}_{1}$. Additionally the linear absorption measured by Fourier transform spectroscopy, as described by Orlita et al. in ref 16 is shown. One can clearly see that the resonance of the $\chi^{(3)}$ process is much narrower compared to the linear absorption. As mentioned before, the dependence on the magnetic field of the FWM stems from the nonlinear susceptibility $\chi^{(3)}$. We derive an expression for $\chi^{(3)}$ using the density matrix formalism similarly to refs 8 and 9. Here we only consider transitions which are close to resonance with the pumping fields. By using this approximation, the linear and second-order density matrix elements are solely determined by the pumping field 
$E_{2}$, while the field $E_{1}$ only enters the third-order density matrix elements. For our specific case, i.e. two incident fields with the same linear polarization, and under the assumption of equal line broadening factors $\gamma$ for the different transitions, the surface susceptibility can be written as:

$$
\begin{gathered}
\chi^{(3)}\left(\omega, 2 \vec{k}_{2}-\vec{k}_{1}\right)=\frac{1}{2 \pi l_{c}^{2} \hbar^{3} \epsilon_{0}} \frac{e^{4} v_{f}^{4}}{\omega \omega_{c}^{3}} \frac{i}{\omega+i \gamma-\omega_{c}} \\
\times \frac{1}{2\left(2 \omega+i \gamma-2 \omega_{c}\right)} \frac{\left(\rho_{-1}-\rho_{0}\right)-\left(\rho_{0}-\rho_{1}\right)}{\omega+i \gamma-\omega_{c}} .
\end{gathered}
$$

Here $v_{f}$ is the Fermi velocity in graphene, $\omega_{c}=$ $v_{f} \sqrt{\frac{2 e B}{\hbar}}$ is the resonance frequency, $l_{c}=\sqrt{\frac{\hbar}{e B}}$ the magnetic length, $\rho_{i}$ is the occupation of the $\mathrm{LL}_{i}$ and $\gamma$ is the line broadening. The numerator of the last fraction is in our case approximately -0.04 , because of the small doping. For intrinsic graphene $\chi^{(3)}$ would vanish in this configuration since contributions from the transitions $\mathrm{LL}_{-1} \rightarrow \mathrm{LL}_{0}$ and $\mathrm{LL}_{0} \rightarrow \mathrm{LL}_{1}$ cancel each other. This reflects electron-hole symmetry of intrinsic graphene. The line broadening for the calculation was taken from the linear absorption measurement by applying a Lorentzian fit to the absorption line (see inset in Figure 4), which yielded $\gamma \simeq 3.5 \mathrm{meV}$. Note that this corresponds to a total dephasing time of $\tau=\hbar / \gamma=190 \mathrm{fs}$, assuming a homogeneously broadened line. The fact that the decay of FWM signal was faster than the pulse duration of $4 \mathrm{ps}$ is consistent with this number. The experimental FWM resonance appears to be slightly broader than the calculated one (see Figure 4). This may have two possible reasons. First, eq 2 does not account for saturation effects. Taking them into account will broaden the calculated peak. Second, far away from the resonance the effect of scattered stray light is more dominant, as the pump-probe signal possesses a broader resonance. Consequently, the strength of the experimental FWM signal may be overestimated in the nearly off-resonant case.

Finally we discuss the strength of the $\chi^{(3)}$ process. According to eq 2 one layer of graphene with $\rho_{0}=0.52$ in a magnetic field resonant to the photon energy features a surface susceptibil- ity of $4.9 \cdot 10^{-20} \mathrm{~m}^{3} / \mathrm{V}^{2}$. This corresponds to a bulk susceptibility of $1.6 \cdot 10^{-10} \mathrm{~m}^{2} / \mathrm{V}^{2}$, assuming a layer thickness of $0.3 \mathrm{~nm}$. For comparison with the experiment it is reasonable to select one of the lower excitation data points from Figure 3 a), where the saturation is negligible. From the second point, where the incoming fields are set to $E_{2}=4.6 \mathrm{kV} / \mathrm{cm}$ and $E_{1}=5.9 \mathrm{kV} / \mathrm{cm}$ and $\mathrm{E}_{\mathrm{FWM}}=0.023 \mathrm{kV} / \mathrm{cm}$ is measured, we derive $\chi^{(3)} \sim 9.2 \cdot 10^{-20} \mathrm{~m}^{3} / \mathrm{V}^{2}$ using eq 1 . Note, that the experimental value is not the $\chi^{(3)}$ for one single layer of graphene, as our sample consists of roughly 50 layers. Considering the uncertainty in the experimental determination of $\mathrm{E}_{\mathrm{FWM}}$ experiment and theory are in reasonable agreement. For doped graphene, where the zeroth Landau-level is either completely filled or empty at a magnetic field of several Tesla, a much higher susceptibility is expected, as the numerator in eq 2 will be 25 times higher (i.e. $\chi^{(3)} \sim 10^{-18} \mathrm{~m}^{3} / \mathrm{V}^{2}$ ). Considering the small thickness of graphene this is in fact a strong nonlinearity. The surface susceptibility of Landau-quantized graphene is comparable to the value observed for intersubband transitions in GaAs quantum wells with much larger thickness and $2 \mathrm{D}$ electron density. For example, the intersubband transition at $124 \mathrm{meV}$ in a GaAs/AlGaAs superlattice results in $\chi^{(3)} \sim 1.3 \cdot 10^{-18} \mathrm{~m}^{3} / \mathrm{V}^{2}$, which is three orders of magnitude larger than the intraband nonlinearity due to nonparabolicity of the wells ${ }^{17}$. Even higher third-order nonlinearities $\chi^{(3)} \sim 5 \cdot 10^{-17} \mathrm{~m}^{3} / \mathrm{V}^{2}$ are predicted in the $\mathrm{THz}$ range by utilizing impurity transitions in GaAs quantum well ${ }^{18}$, but have not been verified experimentally, yet.

In summary, the proposed large third-order nonlinearity in Landau-quantized graphene is experimentally demonstrated for the first time. The order of $\chi^{(3)}$, the resonance behavior, and the field dependencies are in a good agreement with theoretical predictions. Landau-quantized graphene represents a system with a strong optical nonlinearity and offers spectral tunability by variation of the magnetic field. These properties are attractive for a variety of mid-infrared nonlinear optical applications such as frequency multiplication, parametric generation, and sum-frequency generation.

Acknowledgement We thank P. Michel and his 
team for their dedicated support. This work was financially supported by the SPP 1459 of the DFG.

\section{References}

(1) Mikhailov, S. A.; Ziegler, K. J. Phys. Condens. Matter 2008, 20, 384204.

(2) Mikhailov, S. A. Phys. Rev. B 2016, 93, 085403.

(3) Hendry, E.; Hale, P. J.; Moger, J.; Savchenko, A. K.; Mikhailov, S. A. Phys. Rev. Lett. 2010, 105, 097401.

(4) Ciesielski, R.; Comin, A.; Handloser, M.; Donkers, K.; Piredda, G.; Lombardo, A.; Ferrari, A. C.; Hartschuh, A. Nano Lett. 2015, 15, 4968.

(5) Rao, S. M.; Lyons, A.; Roger, T.; Clerici, M.; Zheludev, N. I.; Faccio, D. Sci. Rep. 2015, 5, 15399.

(6) Woerner, M.; Kuehn, W.; Bowlan, P.; Reimann, K.; Elsaesser, T. New J. Phys. 2013, 15, 025039.

(7) Goerbig, M. O. Rev. Mod. Phys. 2011, 83, 1193.

(8) Yao, X.; Belyanin, A. Phys. Rev. Lett. 2012, $108,255503$.

(9) Yao, X.; Belyanin, A. J. Phys.: Condens. Matter 2013, 25, 054203.

(10) Berger, C.; Song, Z.; Li, X.; Wu, X.; Brown, N.; Naud, C.; Mayou, D.; Li, T.; Hass, J.; Marchenkov, A. N.; Conrad, E. H.; First, P. N.; de Heer, W. A. Science 2006, $312,1191$.

(11) Sun, D.; Divin, C. J.; Berger, C.; de Heer, W. A.; First, P. N.; Norris, T. B. Phys. Rev. Lett. 2010, 104, 136802.

(12) Mittendorff, M.; Orlita, M.; Potemski, M.; Berger, C.; de Heer, W. A.; Schneider, H.; Helm, M.; Winnerl, S. New J. Phys. 2014, $16,123021$.
(13) Plochocka, P.; Kossacki, P.; Golnik, A.; Kazimierczuk, T.; Berger, C.; de Heer, W. A.; Potemski, M. Phys. Rev. B 2009, 80, 245415.

(14) Wendler, F.; Knorr, A.; Malić, E. Nature Commun. 2014, 5, 4839.

(15) Mittendorff, M.; Wendler, F.; Malic, E.; Knorr, A.; Orlita, M.; Potemski, M.; Berger, C.; de Heer, W. A.; Schneider, H.; Helm, M.; Winnerl, S. Nature Phys. 2015, 11,75 .

(16) Orlita, M.; Faugeras, C.; Plochocka, P.; Neugebauer, P.; Martinez, G.; Maude, D. K.; Barra, A.-L.; Sprinkle, M.; Berger, C.; de Heer, W. A.; Potemski, M. Phys. Rev. Lett. 2008, 101, 267601.

(17) Walrod, D.; Auyang, S. Y.; Wolff, P. A.; Sugimoto, M. Appl. Phys. Lett. 1991, 59, 2932.

(18) Yldrm, H.; Aslan, B. Semicond. Sci. Technol. 2011, 26, 085017. 
Graphical TOC Entry

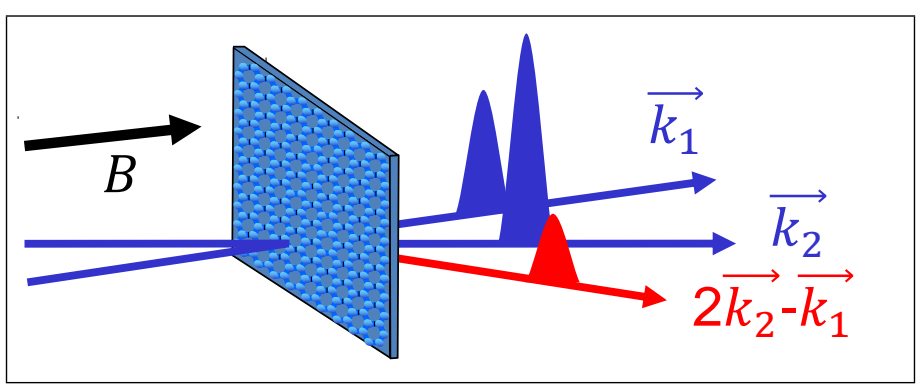

